\title{
Aplicación de estrategias para mejorar la interacción social en niños con trastorno de espectro autista
}

\section{Application of strategies to improve social interaction in children with autism spectrum disorder}

\author{
Pahola Iveth Samaniego Olivas
}

Pahola Iveth Samaniego Olivas. Universidad Pedagógica Nacional del Estado de Chihuahua, Campus Delicias, México. Correo electrónico: samaniego.olivas.pahola.iveth@ gmail.com. ORCID: https://orcid. org/0000-0002-2226-3712.

\section{Resumen}

La presente investigación es de corte cuantitativo, un diseño experimental de caso único. Se realizó con base en la problemática “¿Cuál es el efecto de la aplicación de estrategias para mejorar la interacción social en niños con trastorno de espectro autista?”. Se contó con la participación de un infante de seis años tres meses de edad, y cuatro observadores. El objetivo fue determinar el efecto de la aplicación de estrategias para mejorar la interacción social en niños con trastorno de espectro autista, se empleó el instrumento CSBS-DP para medir comunicación, lenguaje expresivo y simbolización. El instrumento está conformado por 24 reactivos dirigidos a las áreas mencionadas, el valor de la confiabilidad fue de 0.929 (Alfa de Cronbach). El principal resultado fue: las estrategias no tuvieron un impacto significativo en el infante, debido a que la implementación no fue óptima, hubo intervención por más de un individuo, además el tiempo para la aplicación práctica estratégica no fue suficiente como para ser asimilada por el infante.

Palabras clave: Autismo, educación especial, necesidades educativas especiales, equidad educativa, inclusión social.

\begin{abstract}
The present research bears a quantitative approach and a single-case experimental design. It was carried out based on the problem "What is the effect of the application of strategies to improve social interaction in children with autism spectrum disorder?". A six-year, three-month-old infant and four observers participated in the study. The objective was to determine the effect of the application of strategies to improve social interaction in children with autism spectrum disorder. The CSBS-DP instrument was used to measure communication, expressive language and symbolization. The instrument is composed of 24 items directed to the mentioned areas; the reliability value was 0.929 (Cronbach's Alpha). The main result was: the strategies did not have a significant impact on the infant, due to the fact that the implementation was not optimal since there was intervention by more than one individual. In addition, the time for the practical application of the strategies was not enough to be assimilated by the infant.
\end{abstract}

Keywords: Autism, special education, special educational needs, educational equity, social inclusion. 


\section{INTRODUCCIÓN}

En 1911 el doctor Eugen Bleuler empleó por primera vez el término “autismo” para describir un síndrome que se creía que formaba parte de la esquizofrenia, lo presentaba un grupo de niños junto con otros problemas de origen neurológico; dentro del comportamiento observable, la principal característica que se identificó fue lo complejo para crear vínculos de socialización: mostraban un encierro dentro de sí mismos (Balbuena, 2007). En 1943 Leo Kanne afirmó y comprobó que el trastorno autista se encontraba separado y definidamente independiente de la esquizofrenia, lo refirió como "falta de contacto con las personas, ensimismamiento y soledad emocional".

El doctor Hans Asperger realizó un estudio a un grupo de niños que presentaban muchas de las anomalías que habían sido diagnosticados como portadores del síndrome autista: problemas a la hora de socializar y en su comportamiento; la gran diferencia era que sí hablaban. En ese tiempo se pensaba que los niños con autismo sufrían un disturbio emocional o una mengua psíquica, y fue gracias a las investigaciones y descripciones científicas de Kanne y Asperger que se reconoció el autismo como identidad.

Desde los años citados anteriormente el autismo ha sido un fenómeno que ha propulsado con una fuerza magna a investigar y hacer una línea de estudio minuciosa, recibiendo mucho esfuerzo y dedicación por los especialistas de diferentes lugares del mundo que se centran en ella; la definición ha cambiado según la experiencia, las nuevas investigaciones realizadas y aportes y descubrimientos científicos que se han desarrollado para aislar este trastorno de cualquier otro de carácter neuronal, para esto se han determinado síntomas específicos de perfil universal que presentan los infantes portadores de este trastorno. Las características que se delimitaron como específicas del autismo fueron: el retraso o la falta de práctica de comunicación, discapacidad en la habilidad del lenguaje, dificultades en la comprensión, práctica de rituales repetitivos o compulsivos en su forma de expresión corporal.

$\mathrm{Al}$ principio se creyó que el autismo era un problema que se originaba por la forma en que las madres y los padres criaban a sus hijos, actualmente se sabe que las causas surgen por factores tanto genéticos y biológicos como ambientales, mismos que producen una alteración en el sistema nervioso que deja como residuos este tipo de trastornos neuronales que a su vez impiden un desarrollo cognoscitivo en los procesos de comunicación, comportamiento y socialización; se desarrollan movimientos repetitivos, generalmente en las manos y dedos, que el infante siente como una forma de estimulación de sí mismo.

Según Cabrera, "los trastornos generalizados del desarrollo afectan a 25.5/10,000 personas. El autismo es el más común de ellos, ya que se da en 10-20/10,000 individuos, con una predominancia general de 4.1 en varones" (2007, p. 209).

Según los resultados de las valoraciones clínicas etiológicas dentro de las investigaciones del trastorno de espectro autista (TEA), se estima que, de la recurrencia en 
los casos detectados por los pronósticos, $20 \%$ de ellos se debe a síndrome o entidad definida, hace referencia a alteraciones en la estructura de los cromosomas como resultado de las anomalías producidas durante la gametogénesis o forma en que se divide el cigoto durante los primeros meses de gestación. En consecuencia, el 80\% restante de los pronósticos se catalogan por ser no sindrómicos, ya que manejan como característica ser resultado de factores ambientales y una genética compleja; combinación de diferentes tipos de segmentos ADN portadores de información para las células y su función, que originan la herencia del trastorno de espectro autista (Cabrera, 2007).

En los últimos 30 años se han realizado estudios en diferentes países europeos, puesto que el TEA ha aumentado significativamente, aproximadamente de cada 100 personas al menos una es portadora de esta anomalía, y la aceleración de la percepción de los casos se origina por diferentes factores, entre otros: el crecimiento de concientización del autismo en especialistas de la salud, padres, educadores y población en general; cambios en el juicio de las características del autismo; posibles diagnósticos a edades tempranas, y resultados de indicadores geográficos y demográficos (AE, 2017-2020).

\section{EL PROBLEMA}

El presente proyecto de investigación se realizó con un infante de seis años cuatro meses de edad, mismo que presenta características del TEA, con la intención de comprender qué técnicas y métodos pueden utilizarse para mejorar la interacción dentro de la sociedad donde se desenvuelve. La interrogante sobre la que se desprende la investigación es: “¿Cuál es el efecto de la aplicación de estrategias para mejorar la interacción social en niños con trastorno de espectro autista?”.

\section{Objetivo}

Aplicar estrategias que puedan mejorar la inclusión e interacción social de un niño con el trastorno de espectro autista dentro de su contexto.

\section{SUSTENTO TEÓRICO}

El autismo no es una enfermedad, sino un trastorno: una serie de condicionamientos, de conductas y comportamientos de una persona, que juntos conforman un determinado cuadro al que no se le puede dar identidad de enfermedad. Se hace referencia a él como trastorno de espectro autista (TEA), ya que varía en grados, tipos y estadios. La dificultad de este cuadro se distingue por medio de diferentes factores que dictan el nivel de deficiencia cognitiva, la gravedad de la sintomatología, el estado de desarrollo en el que se encuentra y la edad cronológica. El término TEA encierra: trastorno autista (TA), trastornos perturbadores del desarrollo no especificados (TPDNE) y síndrome de Asperger (SA). 
Según Hernández, Risquet y León,

Es un síndrome de disfunción neuropsiquiátrica; es decir, se trata de un conjunto de síntomas, y su presencia indica que hay un trastorno en el sistema nervioso central, fundamentalmente en el cerebro, órgano rector de todo el sistema y donde se originan todas las funciones psíquicas superiores del ser humano [2015, p. 179].

Rogel-Ortiz (2005), especialista mexicano, afirma que el autismo no es una enfermedad sino un síndrome clínico, presente desde los primeros meses de vida y que incluye alteraciones en conducta, comunicación verbal y no verbal e interacción social y emocional anómala. Para esto los tratamientos que se emplean pueden dividirse en fármacos y psicopedagógicos, el tratamiento fármaco es sintomático y no existe uno que sea aceptado de forma unánime, aunque el beneficio de estos se vería reflejado en la disminución de ansiedad, impulsividad y agresividad.

Las principales características que conforman el cuadro del trastorno de espectro autista surgen desde los primeros meses de vida, por lo general son conductas observables que permiten ser identificadas desde muy temprana edad. Un portador del TEA maneja deficiencia para reaccionar cuando se le llama por su nombre; actúa de forma indiferente como si no entendiera; no es capaz de señalar algún objeto, en cambio mira de una forma fija cuando algo llama su atención y muy pocas veces existe una expresión facial; se limita a hacer uso de la imaginación, situación que le provoca estrés y frustración por falta de expresión; se mantiene en un estado aislado, opta por estar solo y, al encontrarse en compañía, evita el contacto visual (Balbuena, 2007).

El portador de trastorno autista Asperger muestra intereses en temas muy restringidos; normalmente es indiferente con lo que le rodea pero cuenta con uno o más temas que despiertan su interés al grado de volverse experto en el dominio del mismo; su forma de percibir las cosas es muy diferente a la de una persona regular, situación que ocasiona una reacción negativa y de rechazo hacia algunos sonidos, sabores, texturas, o forma de ver las cosas que le rodean; en ocasiones revela favoritismo por una o más personas; siente invadido su espacio cuando experimenta un contacto físico (Assumpção y Pimentel, 2000).

El autismo se evalúa por niveles que se determinan de diferentes formas: el nivel 1 se caracteriza por el grado o severidad leve, en este nivel se requiere apoyo pero solo en algunas áreas, ya que se maneja con más facilidad la adaptación a distintos contextos educativos, sociales y familiares; el nivel 2 es el nivel intermedio, en el que la gravedad de las alteraciones es más importante ya que los síntomas que se presentan son más amplios y repercute a afectar más áreas; el nivel tres es el más avanzado en deficiencias que alteran y causan anomalías en las áreas motriz, lingüística, social e intelectual, y se requiere de ayuda de especialistas para conseguir el avance en los trastornos que se provocan.

Las principales características que conforman el cuadro del TEA surgen desde los primeros meses de vida, por lo general son conductas observables que permiten 
ser identificadas desde muy temprana edad. Un portador presenta deficiencia para reaccionar cuando se le llama por su nombre; actúa de forma indiferente como si no entendiera; no es capaz de señalar algún objeto, en cambio mira de una forma fija cuando algo llama su atención; muy pocas veces existe una expresión facial; se limita a hacer uso de la imaginación; se mantiene en un estado aislado; opta por estar solo y, al encontrarse en compañía, evita el contacto visual (Balbuena, 2007). La escasa habilidad de comprender las sutilezas que rigen las marcadas expresiones y gestos (comunicación no verbal) es un gran obstáculo para que se genere una buena interacción social, ya que el portador maneja un pensamiento literal (Baña, 2011).

Por otra parte, mantiene una gran dificultad para tratar de entender y ser empático con los sentimientos y situaciones de otras personas, incluso sus familiares cercanos; tiene retraso en el lenguaje verbal, esto provoca problemas para el estímulo de la comunicación; en diversas ocasiones puede hacer uso repetitivo de frases y palabras que no tienen sentido pero es estimulante hacerlo para el individuo; por lo general responde sin sentido a preguntas formuladas por las personas, ya que se le dificulta la comprensión de frases largas o abstractas; su desarrollo se da con base en rutinas, cuando se origina un desajuste o modificación de estas el portador de TEA tiende a mostrar conductas agresivas, ya que no cuenta con las habilidades necesarias para enfrentar cambios (Balbuena, 2007).

La vida de las personas con autismo es demasiado compleja, ya que percibir las discapacidades que este implica a simple vista resulta un tanto difícil, pues las características que portan estas personas no van acompañadas de rasgos físicos sino conductuales y comportamientos retraídos, es por esta razón que se describe como uno de los trastornos más difíciles de diagnosticar para quienes lo portan y de comprender quienes los acompañan en su trayecto de vida. Por tales razones existe dificultad en el proceso del diagnóstico, pues es hasta después de los 18 meses de edad cuando se puede someter a pruebas para detectarlo. El autismo es una entidad diagnóstica de síndrome muy estudiado (pues existen enigmas que aún no se conocen por especialistas y por padres), no de su originalidad sino de la eficiencia que existe en la aplicación de métodos y estrategias en el desarrollo psicopedagógico.

En ocasiones los padres y los mismos médicos se construyen una idea de omisión en la existencia del trastorno, pero, de acuerdo a las conductas manifestadas, siempre hay una incertidumbre de que el niño se desarrolla de una forma anormal según la comparación con otros niños. Este presenta conductas retraídas, indiferencia por el gusto de socializar, dificultad de comunicación e identificación de códigos lingüísticos, y hasta cierto punto una apariencia de comodidad con la soledad.

Las estrategias desarrolladas para el mejoramiento de estas conductas buscan fomentar un ambiente que motive al infante a realizar por lo menos una pequeña participación activa; estas son: a) estimular el desarrollo hacia el aprendizaje que necesita en su contexto y sociedad, b) utilizar en especial estímulos visuales para de- 
sarrollar la memoria y comprensión, c) hacer un acercamiento por lapsos pequeños y prolongados con la finalidad de crear familiarización en contacto con las personas, d) crear motivación evitando experiencias que le hagan sentir de forma despectiva, y e) brindar su espacio y tiempo para crear una zona donde pueda desarrollar su autonomía. Estas estrategias deben llamar la atención del niño e introducir patrones que le permitan seguir un modelo de conducta (García-Hortal, Sahagún-Navarro y Villatoro-Bongiorno, 2017).

\section{DisEÑO METODOLÓGICO}

Por tratarse de un estudio social y buscar comprender el comportamiento del fenómeno en un tiempo y espacio determinados, se trabajó con el paradigma pos-positivista (Guzmán y Alvarado, 2009). El enfoque cuantitativo exige como condición "datos que sean medibles, observables y cuantificables para comprobar y así tener una base de sustento en el análisis estadístico y medición numérica, con la finalidad de establecer un patrón y mediante la interpretación comprobar teorías" (Escamilla, 2008, p. 8).

Por las características específicas del participante, fue empleado el método experimental de un solo sujeto $(\mathrm{N}=1)$, con diseño de inversión A-B-A (propuesto por Barlow y Hersen), de pretest-postest. Se desarrolla con base en el estudio de un solo individuo, mismo que se encuentra bajo observación constante por varios y diferentes periodos de tiempo (no se cuenta con la participación de otro sujeto para realizar comparaciones), con la finalidad de conocer la conducta y comportamientos observables tomados como base; se crean y se aplican diversas técnicas y estrategias de las cuales se espera una transformación y evolución de comportamiento que ayuden a minimizar el problema presentado bajo el objeto de estudio y las variables identificadas (Guzmán y Alvarado, 2009).

En el diseño del presente experimento, la simbología empleada y sus respectivos significados son:

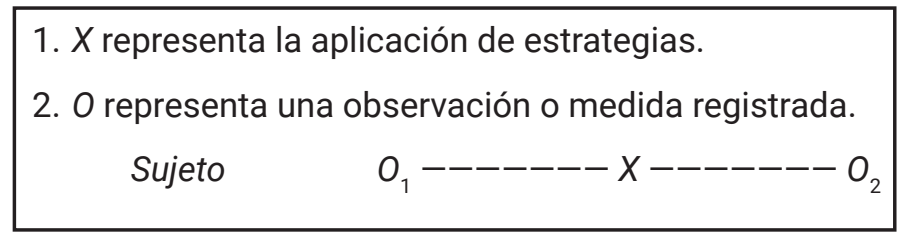

Figura 1. Diseño experimental Fuente: Guzmán y Alvarado, 2009, p. 52.

Para la recolección de datos se utilizó el "Cuestionario del bebé y niño pequeño", o instrumento CSBS-DP, que mide comunicación, lenguaje expresivo y simbolización. Está conformado por 24 reactivos. Fue aplicado en dos momentos por cuatro observadores: antes y después de las estrategias. Estas fueron aplicadas en un lapso 
de tres semanas consecutivas por cinco días (lunes a viernes); se trabajó de manera individual, es decir únicamente con el sujeto participante.

Hipótesis

$\mathrm{H}_{1}$ : La aplicación de estrategias tiene efectos positivos para la mejora de la interacción social en niños con trastorno de espectro autista.

$\mathrm{H}_{0}$ : La aplicación de estrategias tiene efectos nulos para la mejora de la interacción social en niños con trastorno de espectro autista.

\section{Descripción del experimento}

Para trabajar sobre las áreas comunicación, lenguaje expresivo y simbolización, se eligieron siete estrategias, tres buscan desarrollar la comunicación con base en gestos, emociones y miradas (¿Qué estoy mirando?, Caras en la pizarra y Frasco de emociones), dos trabajan el lenguaje expresivo mediante sonidos y emisión de palabras (Completa la canción y Aprende palabras nuevas) y dos amplían la habilidad de la simbolización con estímulo en la comprensión y el uso de objetos (Sigue mi vozy Encesta pelota de trapo).

En ¿Qué estoy mirando? el sujeto encargado de aplicar las estrategias dirige su mirada de una forma fija hacia un objeto y el infante debe saber cuál es el objeto que está mirando. Caras en la pizarra busca ayudar a que el infante pueda expresar sus emociones señalando una o más imágenes colocadas en un pizarrón, que expresan el estado de ánimo. En Frasco de emociones el infante introduce en un frasco tarjetas con imágenes que representan la emoción y estado de ánimo que experimenta en ese momento.

Completa la canción pretende mantener activo el vocabulario usando una canción con letra sencilla en la que el niño repita las palabras y sea capaz de completar palabras o frases que falten en la canción. Aprende palabras nuevas consiste en realizar un diccionario en el cual existan palabras habituales de su contexto y pueda practicarlas en pronunciación. Sigue mi voz trata de hacer posible el razonamiento de indicaciones a actividades sencillas que el infante pueda realizar mediante la instrucción del aplicador de estrategias. Encesta pelota de trapo es una práctica que permite estimular el manejo de objetos y afinar la orientación y puntería del niño al utilizar una caja y una pelota de trapo para lanzar y encestar.

\section{RESULTADOS}

\section{Comunicación}

Mide cómo desarrolla el infante la habilidad de comunicarse con las personas que le rodean. Los subdimensiones son: expresiones de emociones, miradas y gestos.

$\mathrm{Al}$ contestar en la interrogante "¿Puede usted distinguir cuando su niño está contento y cuando está triste?” el 50\% de los observadores afirmó que el niño frecuentemente es capaz de transmitir sus emociones. 


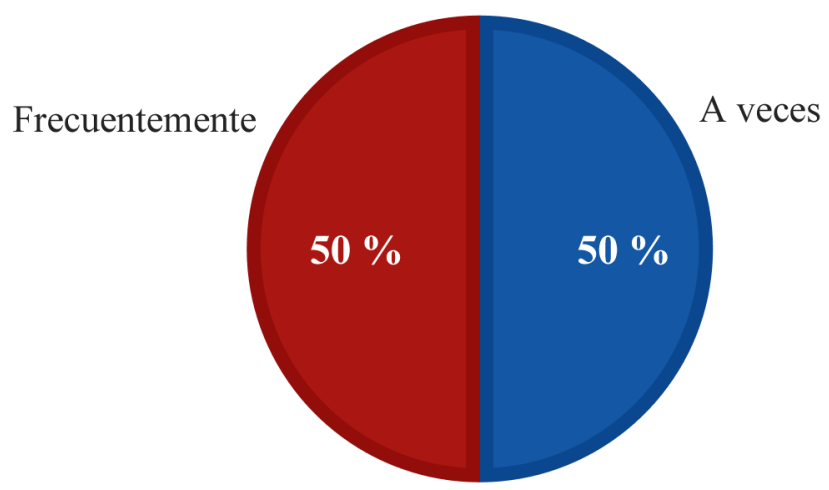

Figura 2. Gráfico de respuestas a la pregunta "¿Puede usted distinguir cuando su niño está contento y cuando está triste?".

Fuente: Elaboración personal.

En la variable "Si usted mira y señala un juguete al otro lado del cuarto, ¿̇u niño o niña se vuelve a verlo?", $50 \%$ de los observadores encontró acciones del niño que muestran que solo a veces este se interesa por mirar objetos o juguetes señalados por otra persona, mientras que el otro 50\% indicó que todavía no existe un interés por parte del niño para voltear a ver.

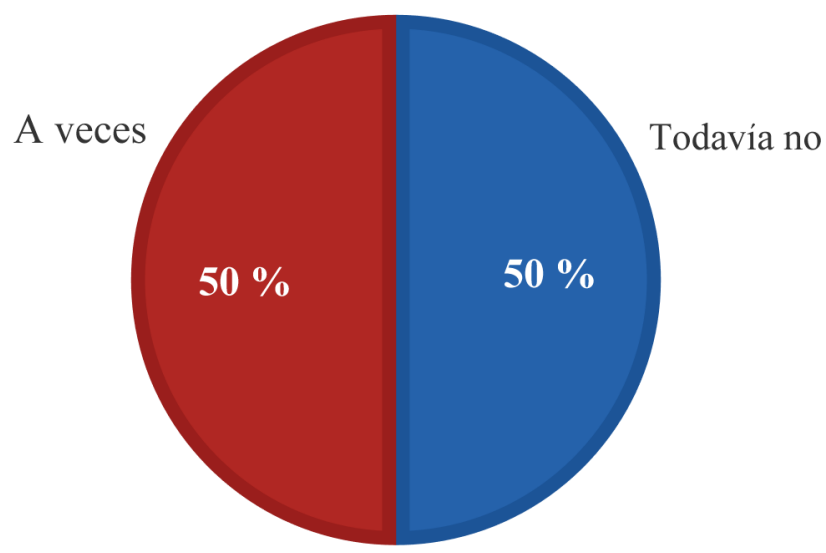

Figura 3. Gráfico de respuestas a la pregunta

"Si usted mira y señala un juguete al otro lado del cuarto, ¿̇su niño o niña se vuelve a verlo?". Fuente: Elaboración personal.

En la interrogante "Si usted no le presta atención, ¿̇u niño o niña trata de llamar su atención?” el 50\% de los observadores notó frecuentemente un comportamiento diferente en el infante para llamar la atención de los que le rodean; el resto afirmó que dichos comportamientos se presentaron solo a veces. 


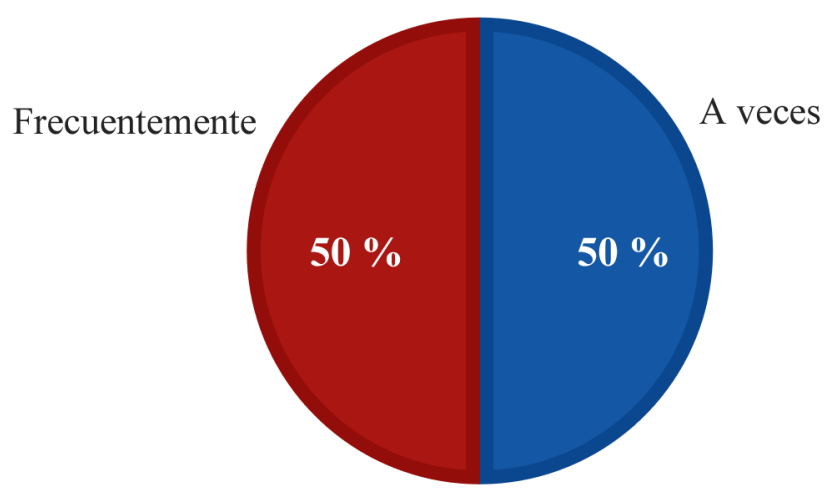

Figura 4. Gráfico de respuestas a la pregunta

"Si usted no le presta atención, ¿̇su niño o niña trata de llamar su atención?".

Fuente: Elaboración personal.

\section{Lenguaje expresivo}

Sonidos y palabras son subdimensiones de la dimensión Lenguaje expresivo, esta mide una de las áreas más afectadas en los portadores de las características del TEA. El instrumento CSBS-DP cuenta con interrogantes que permiten conocer el desarrollo de su vocabulario y uso de sonidos, y permite saber hasta qué nivel se han estimulado e incrementado los mismos con la aplicación de las estrategias.

El 100\% de los observadores solo a veces identificaron conductas o comportamientos en que había un lenguaje expresivo por medio de sonidos y palabras. En comparación con la dimensión de comunicación, el infante tiene más facilidad de comunicación a través de miradas y emociones que por sonidos y palabras.

En la variable "¿Su niño o niña usa sonidos para llamar su atención o pedir ayuda?", el 75\% encontró ocasiones en que el niño exigía atención a las personas por medio de sonidos; por otra parte, el resto de los observadores solo a veces se dieron cuenta cuando el infante expresaba pedir ayuda o necesidad de atención.

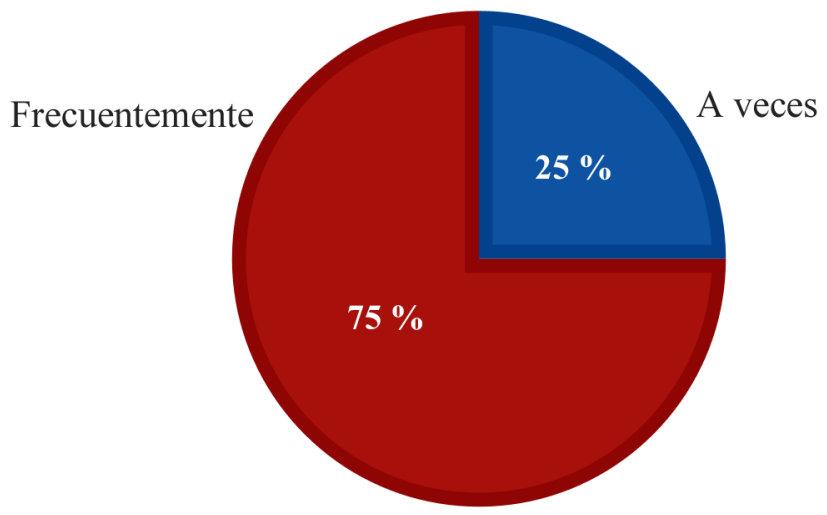

Figura 5. Gráfico de la pregunta

"¿̇u niño o niña usa sonidos para llamar su atención o pedir ayuda?".

Fuente: Elaboración personal. 
En la interrogante " ¿Aproximadamente cuántos sonidos constantes usa su niño o niña: ma, na, ba, da, ga pua, la, ya, sa?”, el 75\% observó que dentro del vocabulario del niño se producen de uno a dos sonidos de los antes mencionados, el resto no identificó ningún tipo de sonido de los señalados.

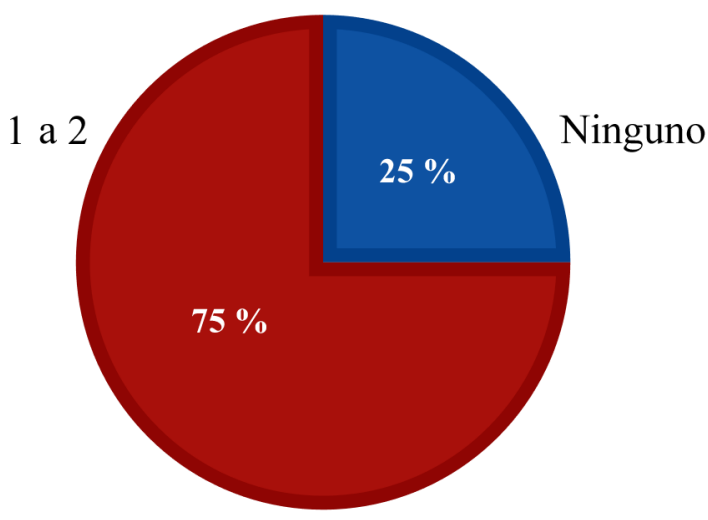

Figura 6. Gráfico de la pregunta

"¿̇Aproximadamente cuántos sonidos constantes usa su niño o niña: ¿̇ma, na, ba, da, ga pua, la, ya, sa?".

Fuente: Elaboración personal.

En la variable “¿Cuántas palabras que usted pueda reconocer usa su niño o niña para indicar algo (como tete por biberón, buba por agua)?”, 75\% afirmó que aún no forma parte del vocabulario del niño palabras para indicar algo. El resto de los observadores afirmó haber escuchado entre una y dos palabras emitidas por el niño para referirse a algo.

\section{Simbolización}

Analiza la capacidad de comprensión que tiene el infante al recibir instrucciones sencillas y complejas, así como medir la habilidad que presenta en el manejo y dominio con el uso correcto de objetos. Los subdimensiones son comprensión y uso de objetos.

El 100\% de los observadores afirma que notó comportamientos en los que solo a veces el infante es capaz de comprender las instrucciones que recibe y le da un uso correcto a los objetos que se le mencionaron anteriormente; falta dominio en cosas de uso diario y comprensión de indicaciones que amerita una respuesta mediante estímulos.

La variable "Cuando llama a su niño o niña por su nombre, ¿responde volviendo la mirada o la cabeza hacia usted?" permite comprobar la asimilación y comprensión del niño. El 100\% de los observadores coincidió en que a veces el infante reacciona al llamado de su nombre, demostrando haber comprendido. 


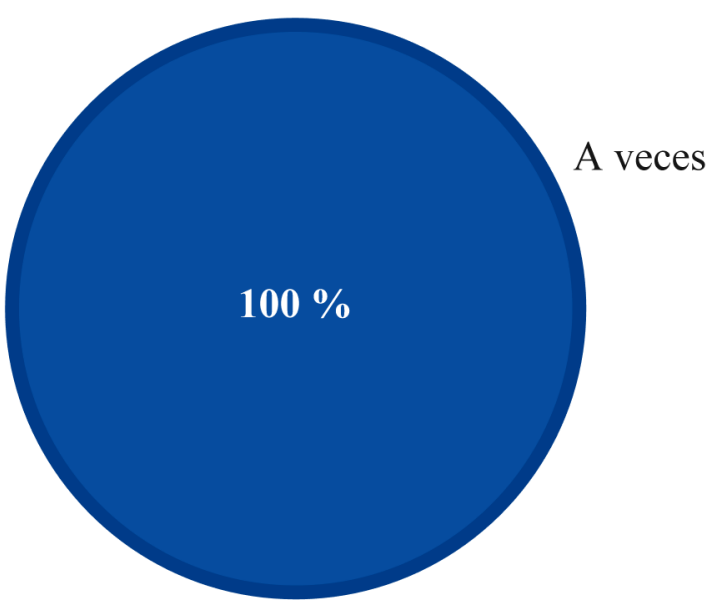

Figura 7. Gráfico de la pregunta

"Cuando llama a su niño o niña por su nombre, żresponde volviendo la mirada o la cabeza hacia usted?". Fuente: Elaboración personal.

La variable “¿Aproximadamente cuántos de los siguientes objetos usa su niño o niña correctamente: taza, biberón, tazón, cuchara, peine, cepillo dental, esponja de baño, pelota, carrito de juguete, teléfono celular?" mide el dominio de uso correcto de objetos. El 100\% de los observadores encontró acciones en el infante que permitieron considerar un uso adecuado de más de ocho objetos de uso diario: el infante tiene la capacidad de hacer buen uso de objetos.

En la variable " ¿Aproximadamente cuántos bloques o aros puede su niño o niña poner uno encima de otro?” el 100\% de los observadores afirmó que fueron más de ocho objetos los que el infante pudo apilar: fue capaz de manejar un alto número de objetos dentro de un mismo espacio, usando sus habilidades para acomodar.

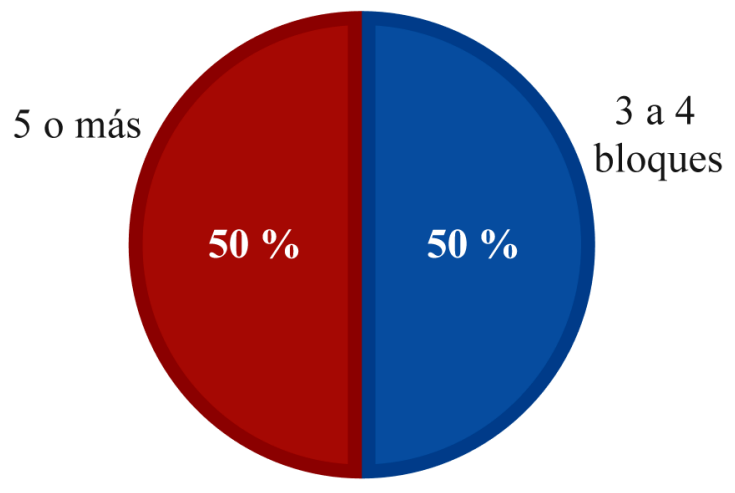

Figura 8. Gráfico de la pregunta

"¿Aproximadamente cuántos bloques o aros puede su niño o niña poner uno encima de otro?".

Fuente: Elaboración personal. 
Con la interrogante “ ¿Con sus juguetes juega como darle de comer a un osito de peluche, arrullar a una muñeca, o poner un muñeco en un carrito?" el 75\% de los observadores percibió que a veces el infante tomaba sus juguetes y realizaba actividades que la mayoría de los niños de su edad hacen, el resto afirma no haber encontrado dichos comportamientos.

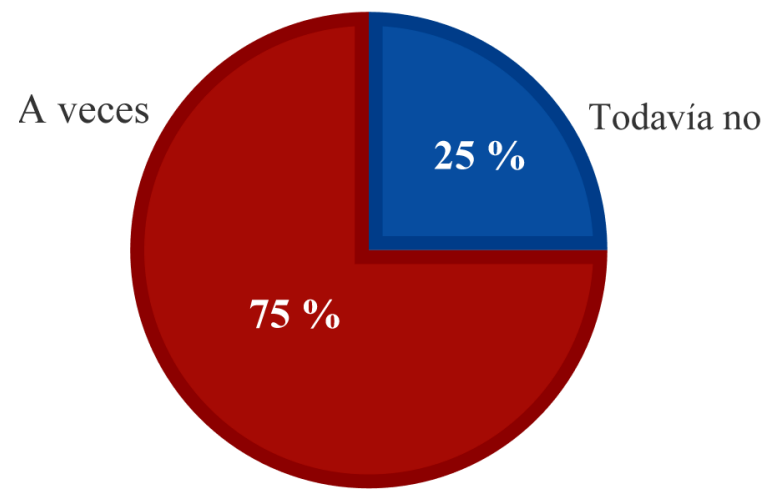

Figura 9. Gráfico de la pregunta "¿Con sus juguetes juega como darle de comer a un osito de peluche, arrullar a una muñeca, o poner un muñeco en un carrito?".

Fuente: Elaboración personal.

\section{Prueba de hipótesis}

Las hipótesis fueron:

$\mathrm{H}_{1}$ : La aplicación de estrategias tiene efectos positivos para la mejora de la interacción social en niños con trastorno de espectro autista.

$\mathrm{H}_{0}$ : La aplicación de estrategias tiene efectos nulos para la mejora de la interacción social en niños con trastorno de espectro autista.

El nivel de medición de la variable corresponde a un tipo cualitativo de alcance ordinal (todavía no, a veces y frecuentemente), los estadísticos para probarla son los noparamétricos. Por la necesidad de comparar los valores de las medianas de dos grupos de datos y por tratarse de datos relacionados (se obtienen de la observación de un mismo sujeto y solo se cuentan con los obtenidos en el pretest y el postest), se empleará el análisis no paramétrico para muestras relacionadas: la prueba de Wilcoxon. El resultado del análisis se presenta en la tabla 1.

Tabla 1. Prueba de hipótesis.

Hipótesis nula

Prueba

Sig.

Decisión

La mediana de las diferencias

Prueba de rangos con signo de Wilcoxon

0.062

Retener la entre pretest y postest es igual a 0 para muestras relacionadas hipótesis nula

Se muestran significaciones asintóticas. El nivel de significación es de 0.05.

Fuente: Elaboración personal. 
El análisis evidencia que las estrategias aplicadas resultan insuficientes para provocar algún cambio en el grado de interrelación social que mantiene el infante con TEA, por esta razón, en esta investigación no se rechaza la hipótesis nula.

\section{DISCUSIÓN}

Con base en los resultados obtenidos en la presente investigación, el efecto de la aplicación de estrategias con intención de mejorar la interacción social de un niño con trastorno de espectro autista (TEA) fue deficiente.

Las condiciones y características de retracción que presenta un niño con TEA en su comportamiento y capacidad de aprendizaje exigen que al emitir una nueva actividad sea con pautas definidas que enmarquen un horario fijo y que el o los aplicadores y observadores emitan la instrucción sin cambios, para que sea capaz de identificar, asimilar y comprender la tarea que debe realizar; una vez que asimila la actividad, se incrementan las posibilidades de que se manifiesten los resultados que se esperan.

Las estrategias eran funcionales, cada diseño se trabajó con base en el área que se pretendía fortalecer, las actividades que comprendían a las mismas, estimulaban el desarrollo cognitivo, implicaban trabajo de lenguaje, socialización y comunicación para crear un vínculo que le permitiera al niño estar en contacto adaptado a la minisociedad que le compone.

$\mathrm{Al}$ aplicar las estrategias, la deficiencia que se detectó fue que la intervención había sido realizada con participación activa de los cuatro observadores; fallar en la implementación de un horario fijo e instrucciones variables, conforme la percepción de cada observador, ocasionó conflicto para el infante a la hora de la asimilación: no se crearon rutinas.

\section{CONCLUSIONES}

1. El síndrome TEA requiere crear rutinas para que el infante sea capaz de asimilar y procesar cualquier tipo de información que le permita un crecimiento óptimo en su desarrollo neuropsiquiátrico, situación que exige un establecimiento de horario y repetición continua en la aplicación de estrategias.

2. El intento de cambio de rutinas, caracterizado por la falta de repetición, así como la ausencia de respeto por el establecimiento de horarios estrictos, impide la generación de nuevos hábitos.

3. Las acciones que se pretende que sean asumidas por el sujeto con TEA deben asumirse tanto por los educadores como por los familiares cercanos, si de verdad se desea el cambio, de otra forma puede ocurrir que se genere confusión en el sujeto, lo que al final deriva en reafirmación de las conductas negativas que se pretende modificar.

Las sugerencias recomendadas para mejorar la efectividad en la aplicación de las estrategias son: 
1. Que el investigador sea quien trabaje y cree un sistema de aplicación de estrategias, con la finalidad de que no exista una mediación externa que genere inconvenientes y resultados no deseados con la intervención del infante.

2. Al aplicar instrumento y práctica de estrategias por un solo sujeto (el investigador) se permitirá un mejor control del experimento.

\section{REFERENCIAS}

AE (2017-2020). Autism Europe. Recuperado de: https:/ /www.autismeurope.org/who-we-are/. Assumpção, F., y Pimentel, A. C. (2000). Autismo infantil. Brazilian Journal of Psychiatry, 22(supl. 2), 37-39. DOI: https://dx.doi.org/10.1590/S1516-44462000000600010.

Balbuena Rivera, F. (2007). Breve revisión histórica del autismo. Revista de la Asociación Española de Neuropsiquiatría, 27(2), 61-81. Recuperado de: http://scielo.isciii.es/scielo.php?script=sci_ arttext\&pid $=$ S0211-57352007000200006\&lng=es\&tlng=es.

Baña, M. (2011). Los trastornos del espectro autista y generales del desarrollo: inclusión social y calidad de vida. Ciencias Psicológicas, 5(2), 183-191. Recuperado de: http://www.scielo. edu.uy/scielo.php?script=sci_arttext\&pid=S1688-42212011000200007\&lng=es\&tlng=es.

Cabrera, D. (2007). Generalidades sobre el autismo. Revista Colombiana de Psiquiatría, 36(1), 208220. Recuperado de: http://www.redalyc.org/articulo.oa?id=80615418016.

Dzul Escamilla, M. (2008). Los enfoques en la investigación cientifica. Recuperado de: https:/ /www. uaeh.edu.mx/docencia/VI_Presentaciones/licenciatura_en_mercadotecnia/fundamentos_de_metodologia_investigacion/PRES39.pdf.

Escamilla, M (2008). Los enfoques en la investigación cientifica. https://www.uaeh.edu.mx/docencia/ VI_Presentaciones/licenciatura_en_mercadotecnia/fundamentos_de_metodologia_investigacion/PRES39.pdf.

García-Hortal, C., Sahagún-Navarro, M., y Villatoro-Bongiorno, K. (2017). Calidad de vida en personas con trastorno del espectro del autismo. Orbis. Revista Científica Ciencias Humanas, 12(36), 65-82. Recuperado de: http://www.redalyc.org/articulo.oa?id=70950101004.

Guzmán Arredondo, A., y Alvarado, J. J. (2009). Fases y operaciones metodológicas en la investigación educativa. Durango, Dgo. México: Asociación de Investigadores en Ciencias de la Educación.

Hernández Rivero, O., Risquet Águila, D., y León Álvarez, M. (2015). Algunas reflexiones sobre el autismo infantil. Medicentro Electrónica, 19(3), 178-181. Recuperado de: http://scielo. sld.cu/scielo.php?script=sci_arttext\&pid=S1029-30432015000300010\&lng=es\&tlng=pt.

Rogel-Ortiz, F. J. (2005). Autismo. Gaceta Médica de México, 141(2), 143-147. Recuperado de: http:/ / www.scielo.org.mx/scielo.php?script=sci_arttext\&pid=S0016-38132005000200009\&lng= es\&tlng $=$ es,

Cómo citar este artículo:

Samaniego Olivas, P. I. (2020). Aplicación de estrategias para mejorar la interacción social en niños con trastorno de espectro autista. RECIE. Revista Electrónica Científica de Investigación Educativa, 5(1), pp. 213-226. doi: doi.org/10.33010/recie.v5i1.989. 